Pacific Journal of Mathematic 


\title{
ON SOME GENERALIZATIONS OF MEIER'S THEOREMS
}

\author{
HIDENOBU YoshidA
}

Dolzhenko formulated the notion of "porosity" for a set, which has been shown to be highly useful in investigations of the theory of cluster sets.

In this paper, we make use of this technique in order to generalize Meier's results [Math. Ann., 147 (1961), 328-344] to the direction including tangential cases.

As an application, we prove a theorem with respect to the existence of Lusin points.

Meier proved remarkable theorems [9, Satz 1 and Satz 2] concerning the boundary behaviors of functions meromorphic in the upper half plane. His proof of Satz 1 depends on Plessner's theorem. But it is proved in [6, Theorem 5], [17, Theorem 3] that a tangential analogue of Plessner's theorem does not hold. So, we must use somewhat different tools to obtain some extensions of Meier's theorem to the direction including tangential cases. On the other hand, his proof of Satz 2 depends on the category-theoretical analogue [9, Theorem 5] of Plessner's theorem, of which an exactly analogous theorem for tangential cases is proved in [3, Theorem 6], [17, Theorem 2].

In this note, we aim to get some generalizations of Meier's theorems, using the concept of the "porosity", introduced by Dolzhenko [5].

Theorem 1 is the fundamental result. As applications of it, we prove in Theorem 2 a tangential analogue of Meier's theorem [9, Satz 1] by making use of the notion of a "pre-Meier point", introduced originally in Dragosh [6], instead of a "Fatou point", and we also prove in Theorem 3 that Meier's theorem [9, Satz 2] has an exactly analogous extension for tangential cases.

As an application of Theorem 2, we prove a theorem with respect to the existence of Lusin points. Finally we put an open question.

1. Notations and definitions. In the following, we denote the unit disc $\{z ;|z|<1\}$ by $D$, the unit circle $\{z ;|z|=1\}$ by $\Gamma$ and the extended $w$-plane by $W$.

Suppose a set $P \subset \Gamma$ and a point $\zeta=e^{i \theta} \in \Gamma$ are given. For a number $\varepsilon>0$, we denote an arc $\left\{e^{i \theta \prime} ; \theta-\varepsilon<\theta^{\prime}<\theta+\varepsilon\right\}$ by $\Gamma(\varepsilon, \zeta)$. Let $\gamma(\zeta, \varepsilon, P)$ be the largest of the lengths of arcs contained in $\Gamma(\varepsilon, \zeta)$ and not intersecting with $P$. The set $P$ is porous at $\zeta$, if

$$
\varlimsup_{\varepsilon \rightarrow 0} \frac{1}{\varepsilon} \gamma(\zeta, \varepsilon, P)>0 \text {. }
$$


A set $P$ is porous on $\Gamma$ if it is so at each $\zeta \in P$. A set which is a countable sum of porous sets on $\Gamma$ is said to be $\sigma$-porous on $\Gamma$.

A $\sigma$-porous set on $\Gamma$ is of the first Baire category on $\Gamma$. A $\sigma$ porous set on $\Gamma$ has no points of density with respect to outer measure (i.e., no points of outer density), hence is of measure 0 (see [12, p. 129, Theorem (10.2)]). But there exists a set, which is of measure 0 and not $\sigma$-porous on $\Gamma$ (see [4, p. 75]).

For $0 \leqq q, 0<\alpha, 0<\delta<1$ and $\zeta=e^{i \theta} \in \Gamma$, we denote a $q$-curve

$$
\left\{z ; \alpha|\arg (z)-\theta|^{q+1}=1-|z|, \quad \arg (z)>\theta\right\}
$$

(or

$$
\left.\left\{z ; \alpha|\arg (z)-\theta|^{q+1}=1-|z|, \quad \arg (z)<\theta\right\}\right)
$$

terminating at $\zeta$ by $t^{+}(\alpha, q)(\zeta)$, called a right $q$-curve (or $t^{-}(\alpha, q)(\zeta)$, called a left $q$-curve). We also denote a part of the $q$-curve $t^{+}(\alpha, q)(\zeta)$ (or $\left.t^{-}(\alpha, q)(\zeta)\right)$,

$$
\left\{z ; \alpha|\arg (z)-\theta|^{q+1}=1-|z|, \quad \arg (z)>\theta,|z|>\delta\right\}
$$

(or

$$
\left.\left\{z ; \alpha|\arg (z)-\theta|^{q+1}=1-|z|, \quad \arg (z)<\theta,|z|>\delta\right\}\right)
$$

by $t^{+}(\alpha, \delta, q)(\zeta)$ (or $\left.t^{-}(\alpha, \delta, q)(\zeta)\right)$. When convenient, we use notations $t(\alpha, q)(\zeta)$ or $t(\alpha, \delta, q)(\zeta)$ without specifying whether it be right or left.

For $0 \leqq q, 0<\alpha<\beta, 0<\delta<1$ and $\zeta \in \Gamma$, we define a right $q$ angle $\nabla^{+}(\alpha, \beta, \delta, q)(\zeta)$ as the open region lying between the $q$-curves $t^{+}(\alpha, q)(\zeta)$ and $t^{+}(\beta, q)(\zeta)$, lying outside the circle $\{z ;|z|=\delta\}$ of its radius $\delta$, sufficiently near 1 . The left $q$-angle at $\zeta$ with parameters $(\alpha, \beta, \delta)$, dedoted by $\nabla^{-}(\alpha, \beta, \delta, q)(\zeta)$, is the reflection of $\nabla^{+}(\alpha, \beta, \delta, q)(\zeta)$ with respect to the radius at $\zeta$. When convenient, we use the shorter notation $\nabla^{+}(q)(\zeta), \nabla^{-}(q)(\zeta)$ and $\nabla(q)(\zeta)$ without specifying whether it be right or left.

For a function $f(z)$ defined in the open unit disc $D$, we define, in the usual manner, the cluster sets at $\zeta$ on the sets $\nabla^{+}(\alpha, \beta, \delta, q)(\zeta)$ (or $\left.\nabla^{-}(\alpha, \beta, \delta, q)(\zeta)\right)$ and denote it by

$$
C_{\nabla^{+}(\alpha, \beta, \delta, q)(\zeta)}(f, \zeta) \quad\left(\text { or } C_{\nabla^{-(\alpha, \beta, \delta, q)(\zeta)}}(f, \zeta)\right) \text {. }
$$

A point $\zeta \in \Gamma$ is said to be a $q$-angular Plessner point of $f(z)$ provided that

$$
C_{\nabla^{+}(q)(\zeta)}(f, \zeta)=W \text { and } C_{\nabla^{-(q)(\zeta)}}(f, \zeta)=W
$$

for each right and left $q$-angle at $\zeta$. The set of all $q$-angular Plessner points of $f(z)$ is denoted by $I_{q}(f)$. 
By an arc at $\zeta=e^{i \theta} \in \Gamma$ we mean a continuous curve $z=z(t)$, $t_{0} \leqq t<1$, in $D$ terminating at $\zeta$, i.e., $\lim _{t \rightarrow 1} z(t)=\zeta$. By an admissible $q$-arc $X_{q}(\zeta)(0 \leqq q)$ at $\zeta$ we mean an arc at $\zeta$ such that the limit

$$
\lim _{t \rightarrow 1} \frac{|\arg (z(t))-\theta|^{q+1}}{1-|z(t)|}=\rho_{X_{q}(\zeta)}>0
$$

exists. The part of an admissible $q$-arc $X_{q}(\zeta)$ lying in $\{z ;|z|>\delta\}$ is denoted as $X_{q}(\delta, \zeta)$.

We denote the cluster set of $f(z)$ at $\zeta$ relative to an admissible $q$-arc $X_{q}(\zeta)$ (or a chord $X(\zeta)$ ) by $C_{X_{q}(\zeta)}(f, \zeta)$ (or $C_{X(\zeta)}(f, \zeta)$ ). We define

$$
\Pi_{\chi_{q}}(f, \zeta)=\bigcap_{X_{q}(\zeta)} C_{X_{q}(\zeta)}(f, \zeta) \quad\left(\text { or } \quad \Pi_{\chi}(f, \zeta)=\bigcap_{X(\zeta)} C_{X(\zeta)}(f, \zeta)\right),
$$

where the intersection is taken over all admissible q-arcs $X_{q}(\zeta)$ (or all chords $X(\zeta))$ at $\zeta$.

We denote by $\Lambda_{q}(f, \zeta)$ (or $\Lambda(f, \zeta)$ ) the set of all values $w \in W$ with the property that $f(z)$ assumes the value $w$ in every $q$-angle $\nabla(q)(\zeta)$ (or every Stolz angle $\Delta(\zeta)$ ) at $\zeta$ arbitrarily close to $\zeta$. We define a q-angular Picard point (or an angular Picard point) of $f(z)$ to be a point $\zeta \in \Gamma$ at which the set $W-\Lambda_{q}(f, \zeta)$ (or $W-\Lambda(f, \zeta)$ ) contains at most two values. We define a $q$-angular alternative point (or an alternative point [2]) of $f(z)$ to be a point $\zeta \in \Gamma$ at which

$$
\Pi_{\chi_{q}}(f, \zeta) \cup \Lambda_{q}(f, \zeta)=W \quad\left(\text { or } \quad \Pi_{\chi}(f, \zeta) \cup \Lambda(f, \zeta)=W\right) .
$$

REMARK 1. For each Stolz angle $\Delta(\zeta)$ (or each 0 -angle $\nabla(0)(\zeta)$ ) at $\zeta$, there exists a 0 -angle $\nabla(0)(\zeta)$ (or a Stolz angle $\Delta(\zeta)$ ) which satisfies $\nabla(0)(\zeta) \subset \Delta(\zeta)$ (or $\Delta(\zeta) \subset \nabla(0)(\zeta)$ ) in the neighborhood of $\zeta$. Hence, for every $\zeta \in \Gamma$ we have $\Lambda(f, \zeta)=\Lambda_{0}(f, \zeta)$ and an angular Picard point of $f(z)$ is equal to a 0 -angular Picard point of $f(z)$. But because of $\Pi_{\chi_{0}}(f, \zeta) \subseteq \Pi_{\chi}(f, \zeta)$, 0-angular alternative point of $f(z)$ is an alternative point of $f(z)$, although the converse seems not necessarily true.

\section{Main results.}

Lemma 1. Let $q, \beta, \gamma, \delta^{\prime}$, and $\mu$ be arbitrarily fixed numbers satisfying $0 \leqq q, 0<\beta, \gamma, \mu, 0<\beta-\mu, 0<\delta^{\prime}<1$. Let $P$ be a subset of $\Gamma$ and $\zeta$ be a point of $\Gamma$. Suppose that the set $P$ is not porous at $\zeta$. Then, if we choose suitable positive numbers $\lambda, \rho$ and $\delta$ satisfying $0<\gamma-\lambda, \delta<1$, for each $z_{0}, z_{0} \in \nabla(\gamma-\lambda, \gamma+\lambda, \delta, q)(\zeta)$, there exists $a$ point $\xi, \xi=\xi\left(z_{0}\right) \in P$ such that $\nabla\left(\beta-\mu, \beta+\mu, \delta^{\prime}, q\right)(\xi) \supset D\left(\rho, z_{0}\right)$, where $D\left(\rho, z_{0}\right)=\left\{z ;\left|z-z_{0}\right|<\rho\left\{R\left(z_{0}\right)\right\}^{q+1}\right\}$ with $R\left(z_{0}\right)=$ the length of the part $t\left(\alpha,\left|z_{0}\right|, q\right)(\zeta)$ of the $q$-curve $t(\alpha, q)(\zeta)$ on which $z_{0}$ lies. 
Proof. Without loss of generality, we may assume that $\zeta=1$. In the following, we suppose that

$$
\nabla(\gamma-\lambda, \gamma+\lambda, \delta, q)(\zeta)=\nabla^{+}(\gamma-\lambda, \gamma+\lambda, \delta, q)(\zeta)
$$

and

$$
\nabla\left(\beta-\mu, \beta+\mu, \delta^{\prime}, q\right)(\xi)=\nabla^{+}\left(\beta-\mu, \beta+\mu, \delta^{\prime}, q\right)(\xi) .
$$

In other cases, we can prove analogously.

To begin with, we will get some estimations which are needed in the subsequent proof.

For arbitrarily fixed numbers $\lambda, \rho$ and $\delta$ satisfying $0<\lambda, 0<\rho<$ $\gamma-\lambda, 0<\delta<1$, we choose a point $z_{0}=r e^{i \theta}$ in $\nabla^{+}(\gamma-\lambda, \gamma+\lambda, \delta, q)(1)$. If we denote by $t^{+}(\alpha, q)(1)$ the $q$-curve on which $z_{0}$ lies, we obtain the inequality

$$
\gamma-\lambda<\alpha<\gamma+\lambda
$$

In [17], we proved

$$
R\left(z_{0}\right)= \begin{cases}\sqrt{1+\alpha^{2}} \theta+O\left(\theta^{2}\right) & (q=0) \\ \theta+O\left(\theta^{q+1}\right) & (q>0) .\end{cases}
$$

We choose $\psi_{1}=\psi_{1}\left(z_{0}\right)$ (or $\left.\psi_{2}=\psi_{2}\left(z_{0}\right)\right)$ so that the $q$-curve $t^{+}(\beta+\mu, q)\left(e^{i \psi_{1}}\right)$, $e^{i \psi_{1}} \in \Gamma$ (or $t^{+}(\beta-\mu, q)\left(e^{i \psi_{2}}\right), e^{i \psi_{2}} \in \Gamma$ ) is tangent to the $\operatorname{disc} D\left(\rho, z_{0}\right)$ from right (or left). We denote the distance from $z_{0}$ to $t^{+}(\beta-\mu, q)\left(e^{\left.i \psi_{1}\right)}\right.$ (or $t^{+}(\beta+\mu, q)\left(e^{i \psi_{2}}\right)$ ) by $h_{1}\left(z_{0}\right)$ (or $\left.h_{2}\left(z_{0}\right)\right)$. The $q$-curve, which passes through $z_{o}$ and terminates at $e^{i \psi_{1}}$ (or $\left.e^{i \gamma_{2}}\right)$, is denoted by $t^{+}\left(\alpha_{1}, q\right)\left(e^{i \gamma_{1}}\right)$ (or $\left.t^{+}\left(\alpha_{2}, q\right)\left(e^{i \gamma_{2}}\right)\right)$. We have

$$
\begin{cases}h_{1}\left(z_{0}\right)= \begin{cases}\frac{\alpha_{1}-(\beta-\mu)}{\sqrt{1+(\beta-\mu)^{2}}}\left(\theta-\psi_{1}\right)+O\left\{\left(\theta-\psi_{1}\right)^{2}\right\} & (q=0) \\ \left\{\alpha_{1}-(\beta-\mu)\right\}\left(\theta-\psi_{1}\right)^{q+1}+o\left\{\left(\theta-\psi_{1}\right)^{q+1}\right\} & (q>0)\end{cases} \\ h_{2}\left(z_{0}\right)= \begin{cases}\frac{(\beta+\mu)-\alpha_{2}}{\sqrt{1+(\beta+\mu)^{2}}}\left(\theta-\psi_{2}\right)+O\left\{\left(\theta-\psi_{2}\right)^{2}\right\} & (q=0) \\ \left\{(\beta+\mu)-\alpha_{2}\right\}\left(\theta-\psi_{2}\right)^{q+1}+o\left\{\left(\theta-\psi_{2}\right)^{q+1}\right\}, & (q>0)\end{cases} \end{cases}
$$

which are proved in [17]. Since we have $h_{1}\left(z_{0}\right)=h_{2}\left(z_{0}\right)=\rho\left\{R\left(z_{0}\right)\right\}^{q+1}$ by assumption, we obtain

$$
\left\{\begin{array}{ll}
\rho\left\{\sqrt{1+\alpha^{2}} \theta+O\left(\theta^{2}\right)\right\}=\frac{\alpha_{1}-(\beta-\mu)}{\sqrt{1+(\beta-\mu)^{2}}}\left(\theta-\psi_{1}\right)+O\left\{\left(\theta-\psi_{1}\right)^{2}\right\} & \\
\rho\left\{\sqrt{1+\alpha^{2}} \theta+O\left(\theta^{2}\right)\right\}=\frac{(\beta+\mu)-\alpha_{2}}{\sqrt{1+(\beta+\mu)^{2}}}\left(\theta-\psi_{2}\right)+O\left\{\left(\theta-\psi_{2}\right)^{2}\right\} &
\end{array}\right\}(q=0)
$$

Here, if we eliminate $r$ from each of systems of simultaneous equations 


$$
\left\{\begin{array} { l } 
{ 1 - r = \alpha \theta ^ { q + 1 } } \\
{ 1 - r = \alpha _ { 1 } ( \theta - \psi _ { 1 } ) ^ { q + 1 } \quad ( q \geqq 0 ) }
\end{array} \text { or } \quad \left\{\begin{array}{l}
1-r=\alpha \theta^{q+1} \\
1-r=\alpha_{2}\left(\theta-\psi_{2}\right)^{q+1} \quad(q \geqq 0),
\end{array}\right.\right.
$$

we have respectively

(5) $\quad \alpha_{1}=\frac{\alpha \theta^{q+1}}{\left(\theta-\psi_{1}\right)^{q+1}} \quad(q \geqq 0) \quad$ or $\quad \alpha_{2}=\frac{\alpha \theta^{q+1}}{\left(\theta-\psi_{2}\right)^{q+1}} \quad(q \geqq 0)$.

Thus, from (4) and (5), we have

(6)

$$
\left\{\begin{array}{l}
\psi_{1}\left(z_{0}\right)=\left\{1-\frac{\alpha-\rho \sqrt{1+(\beta-\mu)^{2}} \sqrt{1+\alpha^{2}}}{\beta-\mu}+o(1)\right\} \theta \\
\psi_{2}\left(z_{0}\right)=\left\{1-\frac{\alpha+\rho \sqrt{1+(\beta+\mu)^{2}} \sqrt{1+\alpha^{2}}}{\beta+\mu}+o(1)\right\} \theta \\
\psi_{1}\left(z_{0}\right)=\left\{1-\left(\frac{\alpha-\rho}{\beta-\mu}\right)^{1 /(q+1)}+o(1)\right\} \theta \\
\psi_{2}\left(z_{0}\right)=\left\{1-\left(\frac{\alpha+\rho}{\beta+\mu}\right)^{1 /(q+1)}+o(1)\right\} \theta .
\end{array}\right\}(q>0)
$$

And we have

$$
\begin{aligned}
& \psi_{2}\left(z_{0}\right)-\psi_{1}\left(z_{0}\right) \\
& = \begin{cases}{\left[\frac{2 \alpha \mu-\rho \sqrt{1+\alpha^{2}}\left\{(\beta-\mu) \sqrt{1+(\beta+\mu)^{2}}+(\beta+\mu) \sqrt{1+(\beta-\mu)^{2}}\right\}}{\beta^{2}-\mu^{2}}+o(1)\right] \theta} \\
{\left[\frac{\{(\alpha-\rho)(\beta+\mu)\}^{1 /(q+1)}-\{(\alpha+\rho)(\beta-\mu)\}^{1 /(q+1)}}{\left(\beta^{2}-\mu^{2}\right)^{1 /(q+1)}}+o(1)\right] \theta} & (q>0)\end{cases}
\end{aligned}
$$

Hence, from (1) we have

(7) $\psi_{2}\left(z_{0}\right)-\psi_{1}\left(z_{0}\right) \geqq K\left(z_{0}\right)$

$$
= \begin{cases}{\left[\frac{2(\gamma-\lambda) \mu-\rho \sqrt{1+(\gamma+\lambda)^{2}}\left\{(\beta-\mu) \sqrt{1+(\beta+\mu)^{2}}+(\beta+\mu) \sqrt{\left.1+(\beta-\mu)^{2}\right\}}\right.}{\beta^{2}-\mu^{2}}\right.} & (q=0) \\ +o(1)] \theta & (q>0) \\ {\left[\frac{\{(\gamma-\lambda-\rho)(\beta+\mu)\}^{1 /(q+1)}-\{(\gamma+\lambda+\rho)(\beta-\mu)\}^{1 /(q+1)}}{\left(\beta^{2}-\mu^{2}\right)^{1 /(q+1)}}+o(1)\right] \theta} & (q)\end{cases}
$$

These are the required estimates.

Now, we proceed to the proof of Lemma 1.

First, we choose $\lambda$ satisfying

$$
\begin{cases}2(\gamma-\lambda) \mu>0 & (q=0) \\ \{(\gamma-\lambda)(\beta+\mu)\}^{1 /(q+1)}-\{(\gamma+\lambda)(\beta-\mu)\}^{1 /(q+1)}>0 . & (q>0)\end{cases}
$$

Next, for this $\lambda$, we choose $\rho$ satisfying 


$$
\rho<\gamma-\lambda
$$

and

(8) $M= \begin{cases}2(\gamma-\lambda) \mu-\rho \sqrt{1+(\gamma+\lambda)^{2}}\left\{(\beta+\mu) \sqrt{1+(\beta-\mu)^{2}}\right. & \\ \left.+(\beta-\mu) \sqrt{1+(\beta+\mu)^{2}}\right\}>0 & (q=0) \\ \{(\gamma-\lambda-\rho)(\beta+\mu)\}^{1 /(q+1)}-\{(\gamma+\lambda+\rho)(\beta-\mu)\}^{1 /(q+1)}>0 . & (q>0)\end{cases}$

For this $\rho$, we have

$$
D\left(\rho, z_{0}\right) \subset\{z ;|z|<1\}
$$

for every $z_{0} \in \nabla(\gamma-\lambda, \gamma+\lambda, \delta, q)(1)$, if $\delta$ is sufficiently near 1 . Further, for this $\lambda$ and $\rho$, we choose $\delta$ sufficiently near 1 such that (9) holds and

$$
K\left(z_{0}\right)>0
$$

if $z_{0}$ satisfies $\{z ;|z| \leqq \delta\} \cap D\left(\rho, z_{0}\right)=\varnothing$. Thus, for this $\lambda, \rho$ and $\delta$ we obtain from (7) and (10),

(11) $\psi_{2}\left(z_{0}\right)-\psi_{1}\left(z_{0}\right) \geqq K\left(z_{0}\right)>0$, where $z_{0} \in \nabla^{+}(\gamma-\lambda, \gamma+\lambda, \delta, q)(1)$.

Now we suppose that for $\lambda, \rho$ and $\delta$ chosen above, Lemma 1 was false. Then, there exists a sequence

$$
\left\{z_{n}\right\}, z_{n}=r_{n} e^{i \theta_{n}} \in \nabla^{+}(\gamma-\lambda, r+\lambda, \delta, q)(1), \quad z_{n} \rightarrow 1
$$

such that

$$
\text { the arc }\left(e^{i \psi_{1}\left(z_{n}\right)}, e^{i \psi_{2}\left(z_{n}\right)}\right) \text { contains no point of } P \text {. }
$$

And from (11) we have

$$
\psi_{2}\left(z_{n}\right)-\psi_{1}\left(z_{n}\right) \geqq K\left(z_{n}\right)>0
$$

If we set

$$
\varepsilon_{n}=\max \left\{\left|\psi_{1}\left(z_{n}\right)\right|,\left|\psi_{2}\left(z_{n}\right)\right|\right\} \text {, }
$$

we have from (1) and (6)

$$
\varepsilon_{n} \leqq \begin{cases}\left\{1+\frac{\gamma+\lambda+\rho \sqrt{1+(\beta+\mu)^{2}} \sqrt{1+(\gamma+\lambda)^{2}}}{\beta-\mu}+o(1)\right\} \theta_{n} & (q=0) \\ \left\{1+\left(\frac{\gamma+\lambda+\rho}{\beta-\mu}\right)^{1 /(q+1)}+o(1)\right\} \theta_{n} & (q>0)\end{cases}
$$

and, from (12) and (13)

$$
\gamma\left(1, \varepsilon_{n}, P\right) \geqq \psi_{2}\left(z_{n}\right)-\psi_{1}\left(z_{n}\right) \geqq K\left(z_{n}\right)>0 .
$$

Thus, from $\varepsilon_{n} \rightarrow 0(n \rightarrow \infty)$, we have by (8) and (15), 


$$
\varlimsup_{\varepsilon \rightarrow 0} \frac{\gamma(1, \varepsilon, P)}{\varepsilon} \geqq \varlimsup_{n \rightarrow \infty} \frac{K\left(z_{n}\right)}{\varepsilon_{n}} \geqq \frac{M}{\left(\beta^{2}-\mu^{2}\right)^{1 /(q+1)} \omega}>0, \quad(q \geqq 0)
$$

where

$$
\omega= \begin{cases}1+\frac{\gamma+\lambda+\rho \sqrt{1+(\beta+\mu)^{2}} \sqrt{1+(\gamma+\lambda)^{2}}}{\beta-\mu} & (q=0) \\ 1+\left(\frac{\gamma+\lambda+\delta}{\beta-\mu}\right)^{1 /(q+1)} . & (q>0)\end{cases}
$$

This contradicts the assumption that the set $P$ is not porous at $\zeta=1$.

Lemma 2. (See [17, Lemma 2].) Let $\alpha, \lambda$ be numbers satisfying $0<\lambda<\alpha$ and $z_{0}$ be a point in the q-angle $\nabla^{+}(\alpha-\lambda / 2, \alpha+\lambda / 2, \delta, q)(\zeta)$. We denote the distance from $z_{0}$ to $t^{+}(\alpha-\lambda, q)(\zeta)$ (or $t^{+}(\alpha+\lambda, q)(\zeta)$ ) by $h_{1}\left(z_{0}\right)$ (or $\left.h_{2}\left(z_{0}\right)\right)$. If $z_{0}$ lies on a q-curve $t^{+}(\gamma, q)(\zeta)$, put $R\left(z_{0}\right)=$ the length of $t^{+}\left(\gamma,\left|z_{0}\right|, q\right)(\zeta)$. We set

$$
h\left(z_{0}\right)=\min (\text { or } \max )\left\{h_{1}\left(z_{0}\right), h_{2}\left(z_{0}\right)\right\} .
$$

Then we have

$$
K_{n}\left(\text { or } K_{x}\right) \leqq \frac{h\left(z_{0}\right)}{\left\{R\left(z_{0}\right)\right\}^{q+1}} \leqq K_{n}^{\prime}\left(\text { or } K_{x}^{\prime}\right),
$$

where constants $K_{n}$ and $K_{n}^{\prime}$ (or $K_{x}$ and $K_{x}^{\prime}$ ) depend on $\alpha, \lambda$, $\delta$ and satisfy

$$
\lim _{\substack{\lambda \rightarrow 0 \\ \delta \rightarrow 1}} K_{n}=0, \quad \lim _{\substack{\lambda \rightarrow 0 \\ \delta \rightarrow 1}} K_{n}^{\prime}=0 \quad \text { (or } \quad \lim _{\substack{\lambda \rightarrow 0 \\ \delta \rightarrow 1}} K_{x}=0, \lim _{\substack{\lambda \rightarrow 0 \\ \delta \rightarrow 1}} K_{x}^{\prime}=0 \text { ). }
$$

THeorem 1. If $f(z)$ is meromorphic in $D$ and $q \geqq 0$, then except on a $\sigma$-porous set on $\Gamma$, every point of $I_{q}(f)$ is a q-angular Picard point of $f(z)$ or a q-angular alternative point of $f(z)$.

REMARK 2. In the proof of [9, Satz 1], Meier proved the analogous result of this theorem in the case $q=0$ for a function $f(z)$ meromorphic in the upper half plane. But, since his proof is based on an "alternative point" of $f(z)$ in stead of a " 0 -angular alternative point" of $f(z)$, Remark 1 shows that Theorem 1 in the case $q=0$ is sharper than Meier's. Furthermore, I think it is remarkable that, as the exceptional set, the set-theoretical notion " $\sigma$-porosity" is used in stead of "Lebesgue measure 0".

Proof. The fundamental ideas of the following proof go back to Meier [9, Satz 1]. We denote by $E$ the set of points of $\Gamma$, which 
are neither $q$-angular Picard points of $f(z)$ nor $q$-angular alternataive points of $f(z)$. Then, for each point $\zeta \in E$, there exist three different values $a_{\zeta}^{1}, a_{\zeta}^{2}, a_{\zeta}^{3} \in W$, an admissible $q$-arc $X_{q}(\zeta)$ and three $q$-angles $\nabla^{1}(q)(\zeta), \nabla^{2}(q)(\zeta), \nabla^{3}(q)(\zeta)$, which satisfy $a_{\zeta}^{1} \notin C_{X_{q}(\zeta)}(f, \zeta)$ and $f(z) \neq a_{\zeta}^{t}$ for $z \in \nabla^{t}(q)(\zeta)(t=1,2,3)$.

We denote by $F$ the set of points of $E$, at which the above three values $a_{\zeta}^{1}, a_{\zeta}^{2}, a_{\zeta}^{3}$ are finite.

In the following, we will prove that except on a $\sigma$-porous set on $\Gamma$, every point of the set $F$ is not a $q$-angular Plessner point of $f(z)$. For the set $E-F$, we can prove the same conclusion without difficulty.

Let $\left\{c_{i}\right\}_{i=1}^{\infty}$ be a sequence consisting of all complex numbers, which lie dense in the sphere $W$, with rational real parts and imaginary parts. Let $\left\{\beta_{i}\right\}_{i=1}^{\infty}$ be a sequence of all rational numbers satisfying $0<\beta_{i}$.

For positive integers $m_{1}, m_{2}, m_{3}, n_{1}, n_{2}, n_{3}, k$ satisfying the inequality

$$
\left|c_{m_{i}}-c_{m_{j}}\right|>\frac{100}{k} \quad(i \neq j, i, j=1,2,3),
$$

we define $P\left(m_{1}, m_{2}, m_{3}, n_{1}, n_{2}, n_{3}, k\right)$ as the set of points $\zeta$ of $F$, at which the following conditions are satisfied:

$$
\left|f(z)-c_{m_{1}}\right|>\frac{10}{k} \text { for } z \in X_{q}\left(1-\frac{1}{k}, \zeta\right),
$$

$$
\begin{gathered}
f(z) \neq a_{\zeta}^{t} \quad \text { for } \quad z \in \nabla\left(\beta_{n_{t}}-\frac{1}{k}, \beta_{n_{t}}+\frac{1}{k}, 1-\frac{1}{k}, q\right)(\zeta) \quad(t=1,2,3) \\
\left|a_{\zeta}^{t}-c_{m_{t}}\right|<\frac{1}{k} \quad(t=1,2,3)
\end{gathered}
$$

Then we have (see Meier [9, p. 332-333])

$$
F=\sum_{m_{1}, m_{2}, m_{3}, n_{1}, n_{2}, n_{3}, k} P\left(m_{1}, m_{2}, m_{3}, n_{1}, n_{2}, n_{3}, k\right) .
$$

We define $P^{*}\left(m_{1}, m_{2}, m_{3}, n_{1}, n_{2}, n_{3}, k\right)$ as the set of points of $P\left(m_{1}, m_{2}, m_{3}, n_{1}, n_{2}, n_{3}, k\right)$ at which $P\left(m_{1}, m_{2}, m_{3}, n_{1}, n_{2}, n_{3}, k\right)$ is not porous. Then, at every point of the set $P\left(m_{1}, m_{2}, m_{3}, n_{1}, n_{2}, n_{3}, k\right)-$ $P^{*}\left(m_{1}, m_{2}, m_{3}, n_{1}, n_{2}, n_{3}, k\right)$, the set $P\left(m_{1}, m_{2}, m_{3}, n_{1}, n_{2}, n_{3}, k\right)$ is porous, and the set $P\left(m_{1}, m_{2}, m_{3}, n_{1}, n_{2}, n_{3}, k\right)-P^{*}\left(m_{1}, m_{2}, m_{3}, n_{1}, n_{2}, n_{3}, k\right)$ is porous, too. Therefore, the set $P\left(m_{1}, m_{2}, m_{3}, n_{1}, n_{2}, n_{3}, k\right)-P^{*}\left(m_{1}, m_{2}, m_{3}, n_{1}, n_{2}, n_{3}, k\right)$ is a porous set on $\Gamma$.

We will show that every point of the set $P^{*}\left(m_{1}, m_{2}, m_{3}, n_{1}, n_{2}, n_{3}, k\right)$ is not a $q$-angular Plessner point of $f(z)$. In the following, we set 
$P\left(m_{1}, m_{2}, m_{3}, n_{1}, n_{2}, n_{3}, k\right)=P$ and $P^{*}\left(m_{1}, m_{2}, m_{3}, n_{1}, n_{2}, n_{3}, k\right)=P^{*}$.

Let $\zeta \in P^{*}$. For each $t, t=1,2,3$, we set $\beta=\beta_{n_{t}}, \gamma=\rho_{x_{q}(\xi)}, \delta^{\prime}=$ $1-1 / k$ and $\mu=1 / k$ in Lemma 1 , then from Lemma 1 , we can choose positive numbers $\lambda_{t}, \rho_{t}$ and $\delta_{t}\left(0<\delta_{t}<1\right)$ such that for each $z_{0}, z_{0} \in$ $\nabla\left(\rho_{X_{q}(\zeta)}-\lambda_{t}, \rho_{X_{q}(\zeta)}+\lambda_{t}, \hat{o}_{t}, q\right)(\zeta)$, we can find a point $\xi_{t}, \xi_{t}=\xi_{t}\left(z_{0}\right) \in P$ satisfying

$$
\nabla\left(\beta_{n_{t}}-\frac{1}{k}, \beta_{n_{t}}+\frac{1}{k}, 1-\frac{1}{k}, q\right)\left(\xi_{t}\right) \supset D\left(\rho_{t}, z_{0}\right) \quad(t=1,2,3) .
$$

We set

$$
\lambda_{4}=\min \left(\lambda_{1}, \lambda_{2}, \lambda_{3}\right), \quad \rho=\min \left(\rho_{1}, \rho_{2}, \rho_{3}\right) \quad \text { and } \quad \delta_{4}=\max \left(\delta_{1}, \delta_{2}, \delta_{3}\right) .
$$

Then, for each $z_{0}, z_{0} \in \Gamma\left(\rho_{X_{q}(\zeta)}-\lambda_{4}, \rho_{X_{q}(\zeta)}+\lambda_{4}, \delta_{4}, q\right)(\zeta)$, we have evidently

$$
\nabla\left(\beta_{n_{t}}-\frac{1}{k}, \beta_{n_{t}}+\frac{1}{k}, 1-\frac{1}{k}, q\right)\left(\xi_{t}\right) \supset D\left(\rho, z_{0}\right) \quad(t=1,2,3) .
$$

Next, for this $\lambda_{4}$, we can choose $\delta_{5}, 1>\delta_{5}>\delta_{4}$, such that

$$
\nabla\left(\rho_{X_{q}(\zeta)}-\lambda_{4}, \rho_{X_{q}(\zeta)}+\lambda_{4}, \delta_{5}, q\right)(\zeta) \supset X_{q}\left(\delta_{5}, \zeta\right) \text {. }
$$

Thus for each $z_{0} \in X_{q}\left(\delta_{5}, \zeta\right)$ we have

$$
\nabla\left(\beta_{n_{t}}-\frac{1}{k}, \beta_{n_{t}}+\frac{1}{k}, 1-\frac{1}{k}, q\right)\left(\xi_{t}\right) \supset D\left(\rho, z_{0}\right) .
$$

And from (1), we have

$$
f(z) \neq \alpha_{\xi_{t}}^{i} \quad(t=1,2,3 ; i=1,2,3) \text { for } z \in D\left(\rho, z_{0}\right) \text { with } z_{0} \in X_{q}\left(\delta_{5}, \zeta\right) \text {. }
$$

Therefore, three different complex numbers $a_{\xi_{1}}^{1}, a_{\hat{\xi}_{2}}^{2}, a_{\hat{\Sigma}_{3}}^{3} \in W$ are not taken by $f(z)$ in $D\left(\rho, z_{0}\right)$ with $z_{0} \in X_{q}\left(\delta_{5}, \zeta\right)$.

Now for each $z_{0} \in X_{q}\left(\delta_{5}, \zeta\right)$, we consider the linear function

$$
z=z_{0}+\left\{R\left(z_{0}\right)\right\}^{q+1} \eta,
$$

which maps the disc $\{\eta ;|\eta|<\rho\}$ of $\eta$-plane into the disc $D\left(\rho, z_{0}\right)$ of $z$-plane. Then the function $F(\eta)=f\left(z_{0}+\left\{R\left(z_{0}\right)\right\}^{q+1} \eta\right)$ is meromorphic in $|\eta|<\rho$ and does not take three values $a_{\xi_{1}}^{1}, a_{\xi_{2}}^{2}, a_{\xi_{3}}^{3} \in W$ there. And we have

$$
\begin{gathered}
\left|c_{m_{i}}-c_{m_{j}}\right|>\frac{100}{k} \quad(i \neq j ; i, j=1,2,3), \\
\left|F(0)-c_{m_{1}}\right|>\frac{10}{k}, \\
F(\eta) \neq a_{\xi_{t}}^{t} \text { for } \quad|\eta|<\rho \quad(t=1,2,3),
\end{gathered}
$$




$$
\left|\alpha_{\xi_{t}}^{t}-c_{m_{t}}\right|<\frac{1}{k} \quad(t=1,2,3) .
$$

By the exactly analogous procedure as in [9, p. 334-335], we can choose a suitable constant $d$, and for this constant $d$ we obtain the result

$$
\left|F(\eta)-c_{m_{1}}\right| \geqq \frac{1}{k} \quad \text { for } \quad|\eta|<d .
$$

Since the linear function $z=z_{0}+\left\{R\left(z_{0}\right)\right\}^{q+1} \eta$ maps the disc $\{\eta ;|\eta|<d\}$ onto the disc $\left\{z ;\left|z-z_{0}\right|<d\left\{R\left(z_{0}\right)\right\}^{q+1}\right\}$, we have from (2)

(3) $\left|f(z)-c_{m_{1}}\right| \geqq \frac{1}{k}$ for $z \in\left\{z ;\left|z-z_{0}\right|<d\left\{R\left(z_{0}\right)\right\}^{q+1}\right\}$ with $z_{0} \in X_{q}\left(\delta_{5}, \zeta\right)$.

Now, in Lemma 2 we set $\alpha=\rho_{X_{q}(\xi)}$ and we choose $\lambda\left(0<\lambda<\lambda_{4}\right)$ and $\delta\left(1>\delta>\delta_{5}\right)$ satisfying $K_{x}^{\prime}<d$. Next we choose $\delta_{6}\left(1>\delta_{6}>\delta\right)$ such that

$$
X_{q}\left(\delta_{6}, \zeta\right) \subset \nabla^{+}\left(\rho_{X_{q}(\zeta)}-\frac{\lambda}{2}, \rho_{X_{q}(\zeta)}+\frac{\lambda}{2}, \delta_{6}, q\right)(\zeta) \cdot
$$

For each $z_{0} \in X_{q}(\delta, \zeta)$, we denote the distance from $z_{0}$ to $t^{+}\left(\rho_{X_{q}(\zeta)}-\lambda, q\right)(\zeta)$ (or $\left.t^{+}\left(\rho_{X_{q}(\zeta)}+\lambda, q\right)(\zeta)\right)$ by $h_{1}\left(z_{0}\right)$ (or $\left.h_{2}\left(z_{0}\right)\right)$ and we set

$$
h\left(z_{0}\right)=\max \left\{h_{1}\left(z_{0}\right), h_{2}\left(z_{0}\right)\right\} \text {. }
$$

Then we have from (4) and Lemma 2

$$
\frac{h\left(z_{0}\right)}{\left\{R\left(z_{0}\right)\right\}^{q+1}} \leqq K_{x}^{\prime}<d, \text { hence } h\left(z_{0}\right)<d\left\{R\left(z_{0}\right)\right\}^{q+1},
$$

for each $z_{0} \in X_{q}\left(\delta_{6}, \zeta\right)$. Thus for $\delta_{7}\left(\delta_{7}>\delta_{6}\right)$ sufficiently near 1 , the $q$ angle $\nabla^{+}\left(\rho_{X_{q}(\zeta)}-\lambda, \rho_{X_{q}}(\zeta)+\lambda, \delta_{7}, q\right)(\zeta)$ is covered by the set

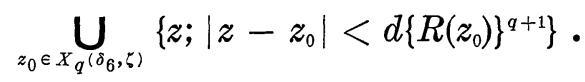

Hence, from (3) we obtain

$$
\left|f(z)-c_{m_{1}}\right| \geqq \frac{1}{k} \quad \text { for } \quad z \in \nabla^{+}\left(\rho_{X_{q}(\zeta)}-\lambda, \rho_{X_{q}(\zeta)}+\lambda, \delta_{7}, q\right)(\zeta),
$$

and this shows that

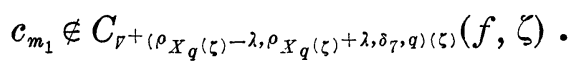

Therefore, $\zeta$ is not a $q$-angular Plessner point of $f(z)$.

Since $\zeta$ is an arbitrary point of $P^{*}$, every point of the set $P^{*}\left(m_{1}, m_{2}, m_{3}, n_{1}, n_{2}, n_{3}, k\right)$ is not a $q$-angular Plessner point of $f(z)$. 
Thus, except on the set

$$
P\left(m_{1}, m_{2}, m_{3}, n_{1}, n_{2}, n_{3}, k\right)-P^{*}\left(m_{1}, m_{2}, m_{3}, n_{1}, n_{2}, n_{3}, k\right),
$$

which is $\sigma$-porous on $\Gamma$, every point of the set $P\left(m_{1}, m_{2}, m_{3}, n_{1}, n_{2}, n_{3}, k\right)$ is not a $q$-angular Plessner point of $f(z)$.

Next, we will obtain a tangential analogue of Satz 1 of Meier [9]. We define

$$
C_{A_{q}}(f, \zeta)=\bigcup_{\Gamma(q)(\zeta)} C_{\nabla(q)(\zeta)}(f, \zeta),
$$

where the summation is taken over all $q$-angles $\nabla(q)(\zeta)$ with vertex at $\zeta$. A point $\zeta \in \Gamma$ is said to be a q-angular pre-Meier point of $f(z)$ provided

$$
\Pi_{\chi_{q}}(f, \zeta)=C_{A_{q}}(f, \zeta) \varsubsetneqq W .
$$

Lemma 3. (See [17, Theorem 1].) Let $q \geqq 0$ and $f(z)$ be meromorphic in $D$. Then except on a $\sigma$-porous set on $\Gamma$ every point $\zeta \in \Gamma$ is either a q-angular pre-Meier point of $f(z)$ or a q-angular Plessner point of $f(z)$.

Theorem 2. Let $q \geqq 0$ and $f(z)$ be meromorphic in $D$. Then except on a $\sigma$-porous set on $\Gamma$, every point $\zeta \in \Gamma$ is a q-angular preMeier point of $f(z)$ or a q-angular Picard point of $f(z)$ or a q-angular alternative point of $f(z)$.

Proof. From Lemma 3 and Theorem 1, we can easily prove this Theorem 2.

We will obtain a tangential analogue of Satz 2 of Meier [9].

A point $\zeta \in \Gamma$ is said to be a $q$-angular Meier point of $f(z)$ provided

$$
\Pi_{\chi_{q}}(f, \zeta)=C_{D}(f, \zeta) \varsubsetneqq W,
$$

where $C_{D}(f, \zeta)$ denotes the cluster set at $\zeta$ on $D$.

Lemma 4. (See [3, Theorem 6] and [17, Theorem 2].) Let $q \geqq 0$ and $f(z)$ be meromorphic in $D$. Then every point $\zeta \in \Gamma$ is either $a$ q-angular Meier point of $f(z)$ or a q-angular Plessner point of $f(z)$ except on a set of the first Baire category on $\Gamma$.

THEOREM 3. Let $q \geqq 0$ and $f(z)$ be meromorphic in $D$. Then except on a set of the first Baire category on $\Gamma$, every point $\zeta \in \Gamma$ is a q-angular Meier point of $f(z)$ or a q-angular Picard point of $f(z)$ or a q-angular alternative point of $f(z)$. 
Proof. From Lemma 4 and Theorem 1, we can easily prove this Theorem 3.

3. Application. Let $D(r, \zeta), 0<r<1, \zeta \in \Gamma$, denote the disc $\{z ;|z-(1-r) \zeta|<r\}$, internally tangent to the unit circle $\Gamma$ at the point $\zeta \in \Gamma$. A point $\zeta \in \Gamma$ is a Lusin point of a meromorphic function $f(z)$ in $D$, if the Riemannian image of every disc $D(r, \zeta), 0<$ $r<1$, has infinite area. The set of Lusin points will be denoted by $L_{1}(f)$.

A point $\zeta \in \Gamma$ such that

$$
C_{A_{q}}(f, \zeta)=\Pi_{\chi_{q}}(f, \zeta)
$$

will be called a q-angular Lindelöf point of $f(z)$ [1].

THEOREM 4. Let $f(z)$ be meromorphic in $D$. Then except on a $\sigma$-porous set on $\Gamma$, every point $\zeta \in \Gamma$ is either an 1-angular Lindelöf point of $f(z)$ or a Lusin point of $f(z)$.

Proof. Since an 1-angular pre-Meier point of $f(z)$ is an 1-angular Lindelöf point of $f(z)$, the Lemma 3 shows that we have only to make inquiries about the set $I_{1}(f)$. Evidently, an 1-angular Picard point of $f(z)$ is a Lusin point of $f(z)$. So let $E$ be a subset of $I_{1}(f)$ such that every $\zeta \in E$ is neither an 1-angular Lindelöf point of $f(z)$ nor an 1-angular Picard point of $f(z)$. Since for each $\zeta \in E$ there exists an admissible 1 -arc $X_{1}(\zeta)$ such that $C_{X_{1}(\zeta)}(f, \zeta) \varsubsetneqq W, \Pi_{\chi_{1}}(f, \zeta)$ is subtotal for every $\zeta \in E$. And from Theorem 1, except on a $\sigma$-porous set on $\Gamma$, every $\zeta \in E$ is an 1-angular alternative point of $f(z)$. Thus at an 1-angular alternative point $\zeta$ of $f(z)$ in $E, A_{1}(f, \zeta)$ contains an open subset of $W$, hence every disc $D(r, \zeta)$ has an infinite area.

We define the cluster set of $f(z)$ at $\zeta$ on $D(r, \zeta)$ by $C_{D(r, \zeta)}(f, \zeta)$. A point $\zeta \in \Gamma$ is called an oricyclic Fatou point of $f(z)$ with an oricyclic Fatou value $w \in W$ (or an oricyclic Plessner point of $f(z)$ ) provided that

$$
\bigcup_{1>r>0} C_{D(r, \zeta)}(f, \zeta)=\{w\} \quad\left(\text { or } \bigcap_{1>r>0} C_{D(r, \zeta)}(f, \zeta)=W\right) .
$$

The set of oricyclic Fatou points (or oricyclic Plessner points) of $f(z)$ will be denoted by $F_{1}^{*}(f)$ (or $I_{1}^{*}(f)$ ).

We denote, in the usual manner, the set of Fatou points (or Plessner points) of $f(z)$ by $F(f)$ (or $I(f)$ ).

For a meromorphic function $f(z)$ in $D$, we denote by $L(f)$ the set of points $\zeta \in \Gamma$ such that for each $\zeta \in L(f)$ the Riemannian image 
of every Stolz angle $\Delta(\zeta)$ has infinite area.

We remarked in [14] that for a meromorphic function $f(z)$ in $D$, the sets $L^{c}(f)-F(f), F(f)-L^{c}(f), L(f)-I(f)$ and $I(f)-L(f)$ are of measure 0 , where the set $S^{c}$ denotes the complement of a set $S$ with respect to $\Gamma$.

In the tangential case, we want to consider analogous problems.

Since the difference of the sets $I_{1}(f)$ and $I_{1}^{*}(f)$ is at most $\sigma$ porous on $\Gamma$ (therefore, of measure 0 on $\Gamma$ ) by [15, Theorem 2], every point of $I_{1}^{*}(f)$, which is not an 1-angular Lindelöf point of $f(z)$, is a Lusin point of $f(z)$ except on a set of measure 0 on $\Gamma$ by Theorem 4. There exists a bounded holomorphic function $f(z)$ in $D$ such that the set $L_{1}(f)-I_{1}^{*}(f)$ and $F_{1}^{*}(f)-L_{1}^{c}(f)$ are both of measure $2 \pi$ (see [8, Corollary 1], [11, Theorem 4] and [10, Theorem 1]).

Open question. Is it true that for a meromorphic function $f(z)$ in $D$, the set $I_{1}^{*}(f)-L_{1}(f)$ is of measure 0 and also the set $L_{1}^{c}(f)-F_{1}^{*}(f)$ is of measure 0 ?

\section{REFERENCES}

1. J. M. Anderson, Boundary properties of meromorphic functions, Quart. J. Math. Oxford (2), 18 (1967), 103-107.

2. F. Bagemihl, Chordal Limits of Holomorphic Functions as Plessner Points, J. Sci. Hiroshima Univ., 30 (1966), 109-115.

3. - Horocyclic boundary properties of meromorphic functions, Ann. Acad. Sci. Fenn., AI, 385 (1966), 1-18.

4. E. F. Collingwood and A. J. Lohwater, The Theory of Cluster Sets, Camb. Univ. Press, New York, (1966).

5. E. P. Dolzhenko, Boundary properties of arbitrary functions (in Russian), Izv. Acad. Nauk SSSR, 31 (1967), 3-14. English translation: Math. of the USSR-IZVESTIJA, 1 (1967), 1-12.

6. S. Dragosh, Horocyclic cluster sets of functions defined in the unit disc, Nagoya Math. J., 35 (1969), 53-82.

7. J. A. Jenkins, On a problem of Lusin, Michigan Math. J., 3 (1955-1956), 187-189.

8. A. J. Lohwater and G. Piranian, On a conjecture of Lusin, Michigan Math. J., 3 (1955-1956), 63-68.

9. K. Meier, Über die Randwerte der meromorphen Funktionen, Math. Ann., 142 (1961), 328-344.

10. G. Piranian, Construction of functions with prescribed boundary behavior, Ann. Acad. Sci. Fenn., AI, 250 (1958), 1-8.

11. G. Piranian and W. Rudin, Lusin's theorem on areas of conformal maps, Michigan Math. J., 3 (1955-1956), 191-199.

12. S. Saks, Theory of the Integral, Dover Publications, Inc., New York, (1964).

13. T. A. Vessey, Some properties of oricyclic cluster sets, J. d'Anal. Math., 21 (1968), 373-380.

14. H. Yoshida, A remark on Plessner points, J. Fac. Eng. Chiba Univ., 38 (1969), 153-154.

15. - Tangential boundary properties of arbitrary functions in the unit disc, Nagoya Math. J., 46 (1971), (to appear). 
16. H. Yoshida,On Plessner points of meromorphic functions, (to appear).

17. - Tangential boundary behaviors of meromorphic functions in the unit disc, (to appear).

Received March 2, 1972.

Chiba University, Chiba, JapaN 


\section{PACIFIC JOURNAL OF MATHEMATICS}

\section{EDITORS}

D. Gilbarg and J. Milgram

Stanford University

Stanford, California 94305

\author{
R. A. Beaumont \\ University of Washington \\ Seattle, Washington 98105
}

J. DuGUNDJI

Department of Mathematics

University of Southern California

Los Angeles, California 90007

Richard ARENS

University of California

Los Angeles, California 90024

\section{ASSOCIATE EDITORS}
E. F. BECKENBACH
B. H. NeumanN
F. WOLF
K. YosHIDA

\section{SUPPORTING INSTITUTIONS}

UNIVERSITY OF BRITISH COLUMBIA

CALIFORNIA INSTITUTE OF TECHNOLOGY

UNIVERSITY OF CALIFORNIA

MONTANA STATE UNIVERSITY

UNIVERSITY OF NEVADA

NEW MEXICO STATE UNIVERSITY

OREGON STATE UNIVERSITY

UNIVERSITY OF OREGON

OSAKA UNIVERSITY

\author{
UNIVERSITY OF SOUTHERN CALIFORNIA \\ STANFORD UNIVERSITY \\ UNIVERSITY OF TOKYO \\ UNIVERSITY OF UTAH \\ WASHINGTON STATE UNIVERSITY \\ UNIVERSITY OF WASHINGTON
* * * *
AMERICAN MATHEMATICAL SOCIETY \\ NAVAL WEAPONS CENTER
}

The Supporting Institutions listed above contribute to the cost of publication of this Journal, but they are not owners or publishers and have no responsibility for its content or policies.

Mathematical papers intended for publication in the Pacific Journal of Mathematics should be in typed form or offset-reproduced, (not dittoed), double spaced with large margins. Underline Greek letters in red, German in green, and script in blue. The first paragraph or two must be capable of being used separately as a synopsis of the entire paper. The editorial "we" must not be used in the synopsis, and items of the bibliography should not be cited there unless absolutely necessary, in which case they must be identified by author and Journal, rather than by item number. Manuscripts, in duplicate if possible, may be sent to any one of the four editors. Please classify according to the scheme of Math. Rev. Index to Vol. 39. All other communications to the editors should be addressed to the managing editor, Richard Arens, University of California, Los Angeles, California, 90024.

50 reprints are provided free for each article; additional copies may be obtained at cost in multiples of 50 .

The Pacific Journal of Mathematics is issued monthly as of January 1966. Regular subscription rate: $\$ 48.00$ a year (6 Vols., 12 issues). Special rate: $\$ 24.00$ a year to individual members of supporting institutions.

Subscriptions, orders for back numbers, and changes of address should be sent to Pacific Journal of Mathematics, 103 Highland Boulevard, Berkeley, California, 94708.

PUBLISHED BY PACIFIC JOURNAL OF MATHEMATICS, A NON-PROFIT CORPORATION

Printed at Kokusai Bunken Insatsusha (International Academic Printing Co., Ltd.), 270, 3-chome Totsuka-cho, Shinjuku-ku, Tokyo 160, Japan. 


\section{Pacific Journal of Mathematics}

\section{Vol. 46, No. 2 December, 1973}

Christopher Allday, Rational Whitehead products and a spectral sequence of

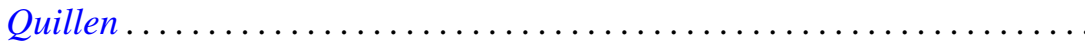

James Edward Arnold, Jr., Attaching Hurewicz fibrations with fiber

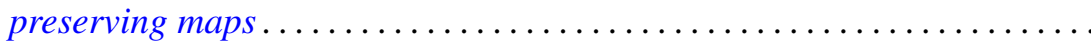

Catherine Bandle and Moshe Marcus, Radial averaging transformations with various metrics.................................

David Wilmot Barnette, A proof of the lower bound conjecture for convex

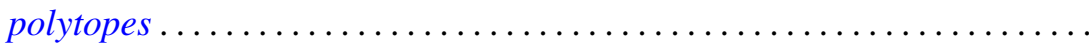

Louis Harvey Blake, Simple extensions of measures and the preservation of

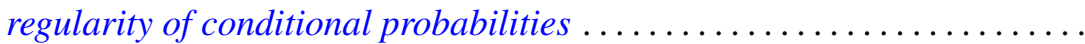

James W. Cannon, New proofs of Bing's approximation theorems for

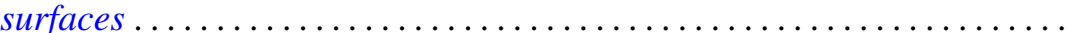

C. D. Feustel and Robert John Gregorac, On realizing HNN groups in

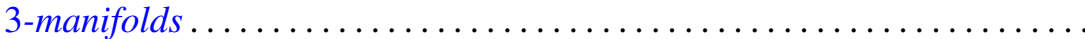

Theodore William Gamelin, Iversen's theorem and fiber algebras . . . . . . . . 389

Daniel H. Gottlieb, The total space of universal fibrations . . . . . . . . . . . .

Yoshimitsu Hasegawa, Integrability theorems for power series expansions of

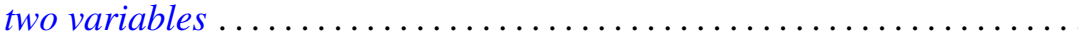

Dean Robert Hickerson, Length of period simple continued fraction expansion of $\sqrt{ } d$

Herbert Meyer Kamowitz, The spectra of endomorphisms of the disc algebra.

Dong S. Kim, Boundedly holomorphic convex domains

Daniel Ralph Lewis, Integral operators on $\mathscr{L}_{p}$-spaces ...

John Eldon Mack, Fields of topological spaces . . . . . . . . .

V. B. Moscatelli, On a problem of completion in bornology

Ellen Elizabeth Reed, Proximity convergence structures. .

Ronald C. Rosier, Dual spaces of certain vector sequence spaces .

Robert A. Rubin, Absolutely torsion-free rings

Leo Sario and Cecilia Wang, Radial quasiharmonic functions . .

James Henry Schmerl, Peano models with many generic classes .

H. J. Schmidt, The $\mathscr{F}$-depth of an $\mathscr{F}$-projector ............

Edward Silverman, Strong quasi-convexity. . . . . . . . . . . . . . . . . 549

Barry Simon, Uniform crossnorms ....................... 555

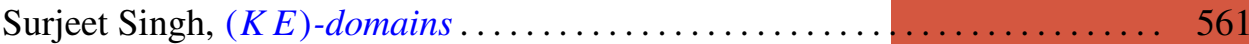

Ted Joe Suffridge, Starlike and convex maps in Banach spaces . . . . . . . . 575

Milton Don Ulmer, $C$-embedded $\Sigma$-spaces . . . . . . . . . . . . . . . . 591

Wolmer Vasconcelos, Conductor, projectivity and injectivity . . . . . . . . . 603 